\title{
In vivo degradation behavior and biocompatibility of Mg-Nd-Zn-Zr alloy at early stage
}

\author{
YONGPING WANG ${ }^{1 *}$, ZHAOJIN ZHU $^{1 *}$, YAOHUA HE $^{1 *}$, YAO JIANG $^{1}$, JIAN ZHANG $^{2}$, \\ JIALIN NIU $^{2}$, LIN MAO $^{2}$ and GUANGYIN YUAN ${ }^{2}$ \\ ${ }^{1}$ Department of Orthopaedics, Sixth People's Hospital of Shanghai Jiao Tong University, Shanghai 200233; \\ ${ }^{2}$ National Engineering Research Center of Light Alloys Net Forming, School of Materials Science \\ and Engineering, Shanghai Jiao Tong University, Shanghai 200240, P.R. China
}

Received August 15, 2011; Accepted September 27, 2011

DOI: $10.3892 /$ ijmm.2011.815

\begin{abstract}
The neotype magnesium alloy, $\mathrm{Mg}-\mathrm{Nd}-\mathrm{Zn}-\mathrm{Zr}$ (NZK) alloy, was implanted into the rabbit femur to investigate its in vivo degradation behavior and biocompatibility. Seventy-two New Zealand white rabbits were randomly divided into the NZK alloy group, titanium alloy group and sham-operated group. Then NZK alloy rods were embedded in the rabbit femur in the NZK alloy group, titanium alloy rods were embedded in the titanium alloy group, and only bone tunnel was established in the sham-operated group. Prior to surgery and at 1, 7, 14, 28 and 56 days after operation, the serum alanine transaminase, creatinine, creatine kinase and magnesium ion concentration were examined in each group. An X-ray of the implanted region was taken at 7, 14, 28 and 56 days after implantation. The pathological changes in heart, liver, kidney and bone from the implant region were examined at 28 and 56 days postoperatively. The degradation behavior of the NZK alloy was observed using scanning electron microscope with an energy dispersive spectroscopy system. There were no significant differences in serum alanine transaminase, creatinine, creatine kinase and magnesium ion concentrations among each group at the same time point $(\mathrm{P}>0.05)$. The histology of heart, liver, kidney and bone from implant region was altered. The results demonstrate that the NZK alloy implanted into the rabbit femur could be absorbed gradually, and that the NZK alloy has excellent biocompatibility in vivo.
\end{abstract}

Correspondence to: Professor Yao Jiang, Department of Orthopaedics, Sixth People's Hospital of Shanghai Jiao Tong University, Shanghai 200233, P.R. China

E-mail: jiangyao195106@163.com

Dr Guangyin Yuan, National Engineering Research Center of Light Alloys Net Forming, School of Materials Science and Engineering, Shanghai Jiao Tong University, Shanghai 200240, P.R. China

*Shared senior authorship

Key words: degradation, biocompatibility, magnesium alloy

\section{Introduction}

Traditionally, materials used for internal fixation in orthopedics are metals such as titanium alloy and stainless steel. The elastic modulus of these metals are greater than that of human bone, thus they can cause a stress shielding effect which can decrease bone strength and delay bone healing. Moreover, these implants may undergo corrosion or abrasion and will release toxic ions or particles into the human body, which result in chronic inflammatory and bone dissolution (1). In addition, the metal materials used for bone fracture fixation are permanent and require removal by an additional surgical procedure, which results in unnecessary morbidity (1-5). Therefore, the development of resorbable metal materials to solve these problems has become the focus of research (6-8).

Magnesium alloys, which are easily corroded in solutions, especially in the presence of chloride ions, and have good biocompatibility have become promising degradable biomaterials, attracting much attention in recent years $(1,2,9,10)$. Previous studies have confirmed that magnesium alloys have good biocompatibility, may promote osteocyte growth and may induce production of osteoblasts and osteocytes (11-15). Owing to suitable mechanical property and good biocompatibility, high specific strength and specific stiffness, degradable magnesium alloys can be utilized in many aspects such as bone repair material, coronary artery stent and porous repair material. However, fast corrosion rates and the appearance of a gas bubble in the physiological environment impose severe limitations which have prevented their further clinical applications. Hence, in order to make use of magnesium alloy feasible for orthopedic implantation, the corrosion rate must be effectively controlled. It is well known that alloying (16) and surface treatment (17) were convenient and effective methods to enhance the corrosion resistance of magnesium. To gain the better corrosion resistance and biocompatibility than that of the commercial magnesium alloys, a novel patent magnesium alloy, Mg-Nd-Zn-Zr (NZK) alloy, has been designed in our laboratory (PRC Patent ZL 201010252357.0). To date, the NZK alloy has not been systematically studied as degradable biomaterial in vitro and in vivo. In the present study, NZK alloy rods were implanted into the rabbit femur so as to analyze the in vivo degradation behavior and biocompatibility at the early 
stage. The results from this study may provide an experimental basis for the in vivo application of NZK alloy and obtain a promising degradable biomaterial for orthopedic applications.

\section{Materials and methods}

Materials and laboratory animals. NZK alloy and titanium alloy were obtained from the National Engineering Research Center of Light Alloys Net Forming, Shanghai Jiao Tong University. The NZK alloy chemical composition is shown in Table I (PRC Patent ZL 201010252357.0). The alloy was fabricated into $\Phi 3 \times 7.5 \mathrm{~mm}$ rods, ground on metallographical emery paper until 1,000 grit, followed by ultrasound wash in ethanol and distilled water. Prior to testing, ethylene oxide was used for sterilization.

A total of 72 clean healthy adult New Zealand white rabbits weighing $2.0-3.0 \mathrm{~kg}$, and an average weight of $2.5 \pm 0.3 \mathrm{~kg}$, were supplied by the Shanghai Jiesijie Lab. Animal Co., Ltd., China [license no. SCXK (hu) 2010-0026]. Thirty-six were male and 36 were female. Protocols were performed in accordance with Guidance Suggestions for the Care and Use of Laboratory Animals, issued by the Ministry of Science and Technology of the People's Republic of China (2006-09-30).

Animal experiments. The seventy-two New Zealand white rabbits were randomly divided into the NZK alloy group, titanium alloy group and the sham-operated group. The New Zealand white rabbits were intramuscularly anesthetized with xylazine (4 mg/kg, Jilin Huamu Animal Health Products Co., Ltd., China) and ketamine (80 mg/kg, Fujian Gutian Medicine Co., Ltd., China). Following fixation, iodophors were utilized to sterilize the skin. Hole sheets were paved. A longitudinal incision about $2 \mathrm{~cm}$ was made in the lateral side of the distal femur. Under sterile conditions, the skin and the subcutaneous tissue were incised to expose the distal femur, and the femur tunnel was established using an electric drill. Then NZK alloy rods were embedded in the femur via femur tunnel in the NZK alloy group, the titanium alloy rods were embedded in the titanium alloy group, and only bone tunnel was established in the sham-operated group (Fig. 1). After saline irrigation, the incision was sutured layer by layer. The rabbits were allowed to move freely in their cages following consciousness, with free access to chow and water. Postoperatively, the animals were received intramuscular injection of penicillin (400 U/day) for three days. The diet, activity, inflammatory reaction and exudation in the incisal opening were observed, and clinical examinations of the femur was performed everyday after the operation until euthanasia of the animals in order to detect the degradation behavior of the NZK alloy implants.

$X$-ray. The region with the implant was photographed using $\mathrm{X}$-rays at 7, 14, 28 and 56 days postoperatively to ensure the correct position of the implant and to be used as a detective method for the degradation of the NZK alloy implants.

Blood analysis. Serum alanine transaminase (ALT), creatinine (CREA), creatine kinase (CK) and magnesium ion concentration (MIC) were examined by an automatic blood biochemistry analyzer (Hitachi 7600-020, Japan) before surgery and at 1, 7, 14, 28 and 56 days after implantation.
Histopathology. Six rabbits from each group were sacrificed by overdose of ketamine at 28 and 56 days postoperatively. The heart, liver, kidney and bone from the implant region were obtained and fixed with $4 \%$ formalin for $24 \mathrm{~h}$, and then dehydrated with gradient ethanol, permeabilized, embedded in paraffin, and sliced into $5 \mu \mathrm{m}$ thick sections. The sections were stained using hematoxylin and eosin (H\&E) staining, and the histological changes in the organs were observed under a microscope.

Scanning electron microscope (SEM) and energy dispersive spectroscopy (EDS). The degradation behavior of the NZK alloy was observed using scanning electron microscope (SEM) with an energy dispersive spectroscopy (EDS) system (Hitachi S-4800, Japan). At 4 and 8 weeks postoperatively, implants were removed from rabbit femur, and rinsed with distilled water and dried in the air. Then the surface morphology was observed by SEM. The products on the surface of the NZK alloy was detected by EDS. At the end, the implants were cleaned by $200 \mathrm{~g} / \mathrm{l}$ chromic acid to remove the corrosion products and the degradation rates were obtained according to the weight loss method (18): corrosion rate $=(\mathrm{K} \times \mathrm{W}) /(\mathrm{A} \times \mathrm{T} \times \mathrm{D})$, where coefficient $\mathrm{K}=8.76 \times 10^{4}$; W, weight loss (g); A, sample area exposed to solution $\left(\mathrm{cm}^{2}\right)$; T, exposure time (h); and D, density of the material $\left(\mathrm{g} / \mathrm{cm}^{3}\right)$.

Statistical analysis. The statistics software SPSS13.0 (SPSS, Inc., Chicago, IL, USA) was used to analyze the data. The difference between the groups was compared using one-way analysis of variance (ANOVA). P-value $<0.05$ indicates statistically significant differences.

\section{Results}

General condition of experimental animals. Of the $72 \mathrm{New}$ Zealand white rabbits obtained a total of 68 were included in the final analysis. Those not included were a rabbit which fractured its surgical region in the ZNK alloy group, 2 rabbits which fractured their surgical region in the sham-operated group, and a rabbit which died of diarrhea in the titanium alloy group after operation. The rabbits in each group grew well, with free activities and normal diet and drinking. No infection, swelling, necrosis, inflammation or flare complicated in local region surrounding the incision.

Radiological examination. As shown in the X-ray photograph (Fig. 2A and B), NZK alloy degradation behavior deepened over time. AT 28 days, the degradation was not noticeable, but the implants became blurred. The size of NZK alloy became small, but did not show obvious degradation at 56 days. The results suggested that NZK alloy might have been absorbed gradually in vivo. However, no significant degradation was visible in the titanium alloy group (Fig. 2C and D).

Results of serology. Changes in ALT, CREA, CK and MIC levels were observed prior to and following surgery in the NZK alloy, titanium alloy and sham-operated groups (Figs. 3-6). No significant differences were observed in ALT, CREA, CK and MIC levels in each group prior to surgery $(\mathrm{P}>0.05)$. There were no significant differences in ALT, CREA and MIC between 
Table I. Chemical composition of the NZK alloy.

Chemical composition (weight \%)

\begin{tabular}{cccccccccc}
\cline { 2 - 9 } & $\mathrm{Mg}$ & $\mathrm{Nd}$ & $\mathrm{Zn}$ & $\mathrm{Zr}$ & $\mathrm{Si}$ & $\mathrm{Ni}$ & $\mathrm{Cu}$ & $\mathrm{Fe}$ & $\mathrm{Al}$ \\
\hline NZK alloy & Balance & 2.5 & 0.2 & 0.5 & 0.0016 & 0.0025 & 0.0195 & 0.1038 & - \\
\hline
\end{tabular}

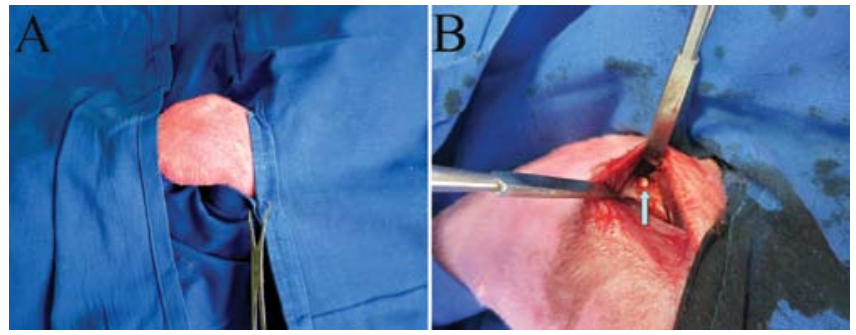

Figure 1. Animal experiment. (A) Preparation for surgery. (B) Implant in the femur of the rabbit.

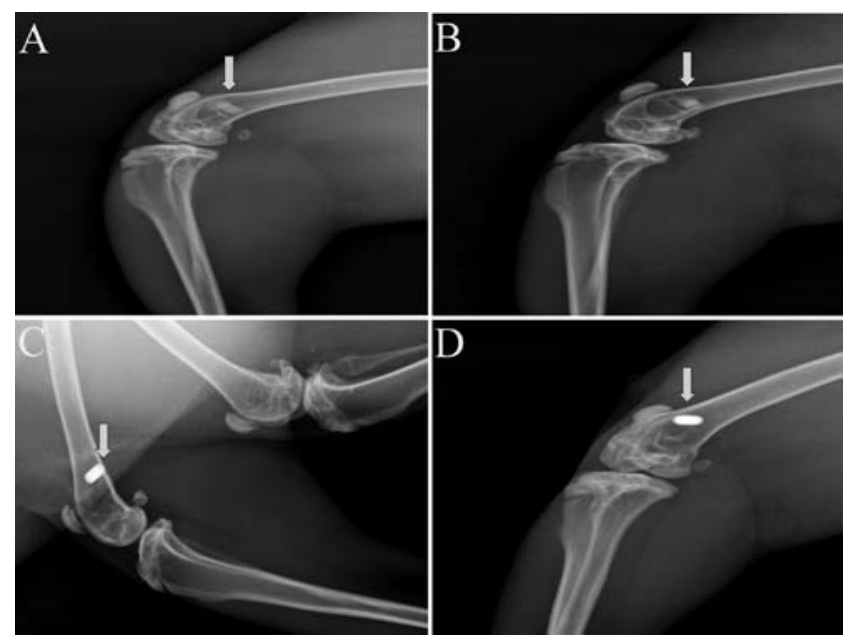

Figure 2. X-ray images of the implant region in the NZK alloy group and titanium alloy group at 28 and 56 days postoperatively. (A) NZK alloy implanted into the rabbit femur 28 days after surgery. (B) The image of the NZK alloy was blurred at 56 days after the surgery. (C and D) Images of the titanium alloy at 28 and 56 days respectively after surgery.

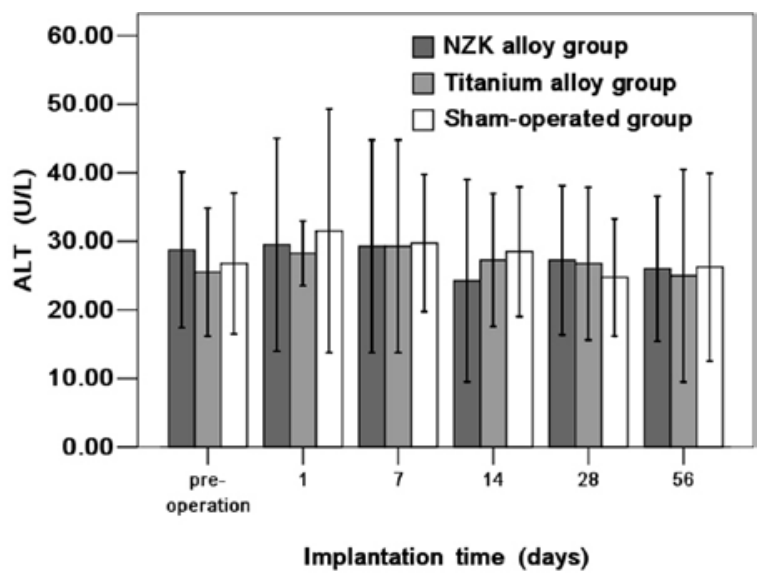

Figure 3. Serum alanine transaminase (ALT) levels before and after implantation in each group.

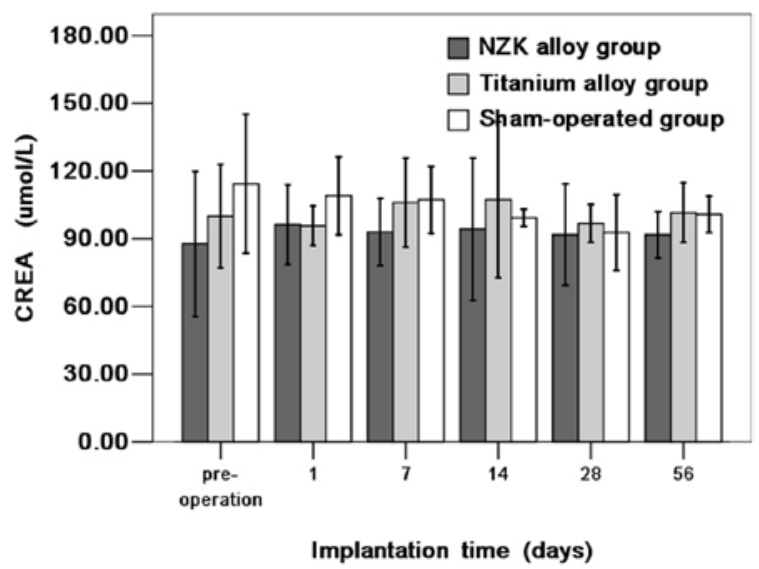

Figure 4. Serum creatinine (CREA) levels before and after implantation in each group.

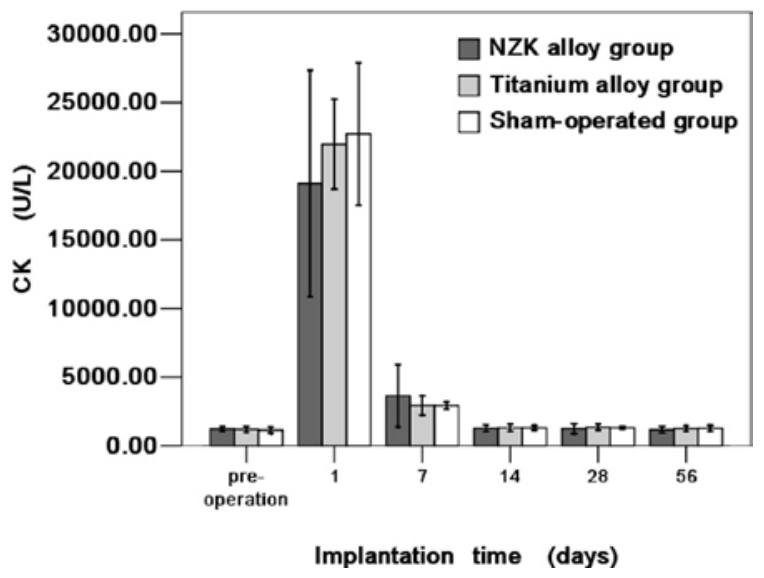

Figure 5. Serum creatine kinase (CK) levels before and after implantation in each group.

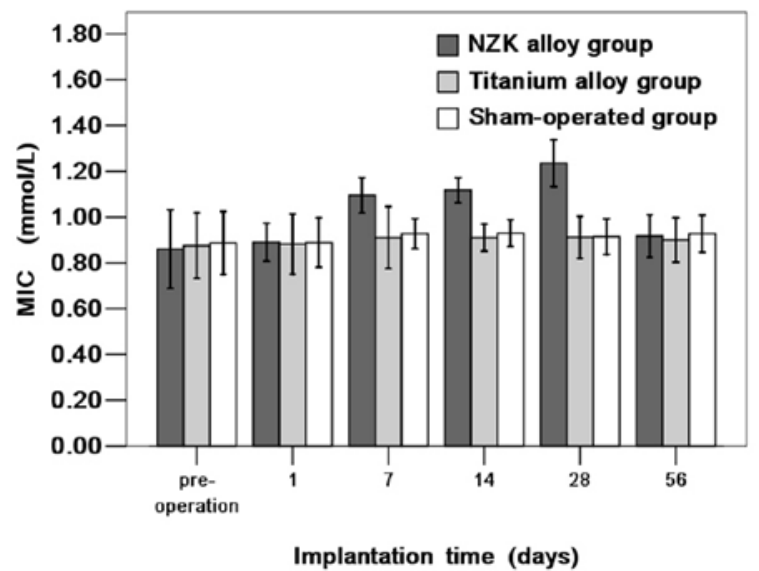

Figure 6. Magnesium ion concentrations (MIC) before and after implantation in each group. 


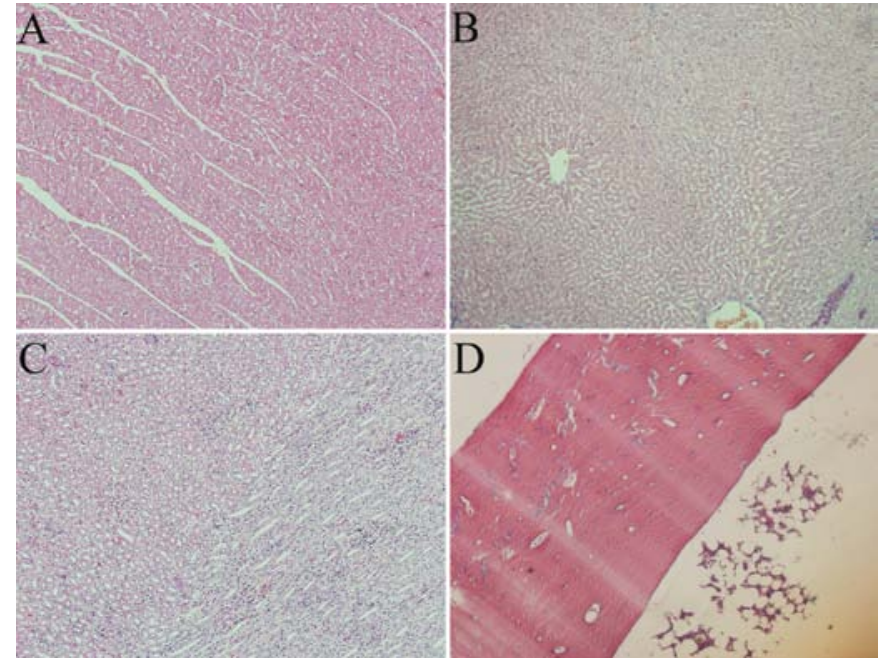

Figure 7. Pathological sections in the NZK alloy group showing normal structure of (A) heart, (B) liver, (C) kidney and (D) bone tissue from the implant region at 4 weeks after the surgery (hematoxylin eosin staining). Magnification, $\mathrm{x} 100$

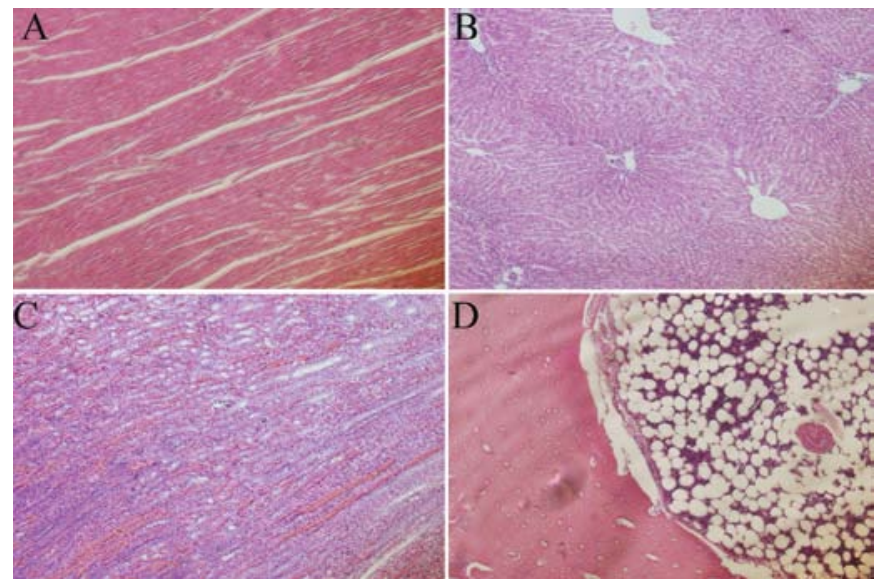

Figure 8. Pathological sections in the NZK alloy group showing normal structure of (A) heart, (B) liver, (C) kidney and (D) bone tissue from the implant region at 8 weeks after the surgery (hematoxylin eosin staining), Magnification, $\mathrm{x} 100$.

the NZK alloy, titanium alloy and sham-operated groups at the same time point following surgery $(\mathrm{P}>0.05)$. On the first day after the surgery, there was a rapid increase of the serum $\mathrm{CK}$ levels $(\mathrm{P}<0.05)$, which returned to the pre-operative levels at 14 days after the surgery. However, no significant differences were determined between the three groups at the same time point $(\mathrm{P}>0.05)$

Histological examination. The New Zealand white rabbits were sacrificed at 28 and 56 days after the surgery and pathological sections were obtained from the heart, liver, kidney, and bone from the implant region and were stained with $\mathrm{H} \& \mathrm{E}$ (Figs. 7 and 8). In the NZK alloy group, the morphology of the myocardium was normal, and did not show substantial changes. Hepatocytes did not present significant swelling or necrosis, and the bile duct did not show obvious expansion or bile siltation. No edema or inflammation was observed in the renal glomerulus. No inflammatory cell infiltration was found in the bone at the implant region. In the titanium alloy group,

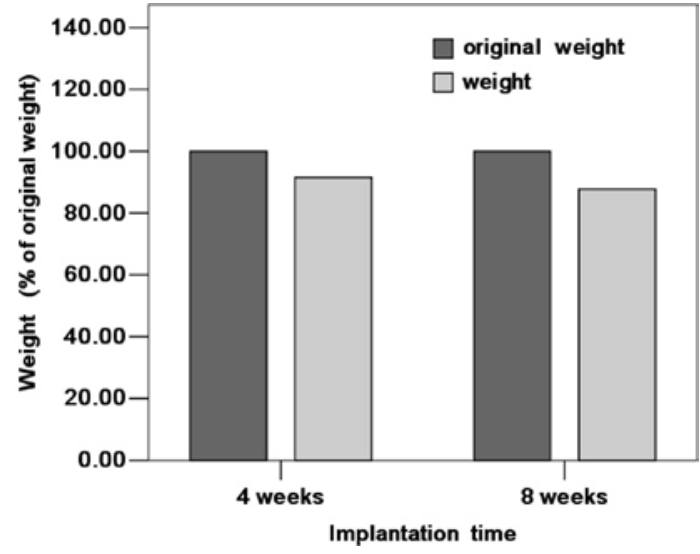

Figure 9. Degradation rates of NZK alloy implanted into the rabbit femur at 28 and 56 days, respectively.

Table II. In vivo degradation rates of NZK alloy.

In vivo degradation rates (mm/year)

\begin{tabular}{lcc} 
& 4 weeks & 8 weeks \\
\hline NZK alloy & $0.66 \pm 0.15$ & $0.48 \pm 0.08$ \\
\hline
\end{tabular}

the structure of the myocardium was not noticeably altered. Hepatocytes were normal, no remarkable inflammation or edema, and the bile duct did not expand. The renal glomerulus exhibited normal morphology, and no inflammatory cell infiltration was detected. The morphology of the bone at the implant region was normal. In the sham-operated group, no inflammation or necrosis was observed in myocardium. Hepatocytes and the hepatic plate arranged normally, and no marked abnormality was determined in the central vein. The renal glomerulus and renal tubule showed normal morphology, without inflammatory cell infiltration. The bone tunnel was healed well in the operation region, and no dramatic inflammatory cell infiltration was observed.

Degradation analysis of ZNK alloy. The NZK alloy was removed from the rabbit femur at 28 and 56 days after the surgery. Its weight was 91.5 and $87.8 \%$ of the original weight at 28 and 56 days respectively, suggesting that 8.5 and $12.2 \%$ of the NZK alloy implants had been absorbed at 28 and 56 days, respectively (Fig. 9). According to the weight loss method, the average degradation rates of the NZK alloy implanted into the rabbit femur were 0.66 and $0.48 \mathrm{~mm} /$ year at 28 and 56 days, respectively (Table II). At visual inspection, the surface of the NZK alloy had lost its original metallic luster, and its edge was not distinct. After drying, the surface was coated with some loose white materials. A layer of off-white degradation products under the SEM was visible on the surface of the NZK alloy (Fig. 10A). The surface was rough with irregular corrosion pits on the NZK alloy when the implants were cleaned by chromic acid to remove the degradation products (Fig. 10B).

EDS analysis showed that the main components of the degradation products were oxygen, carbon, nitrogen, magnesium and phosphorus at 56 days after the surgery (Table III). 


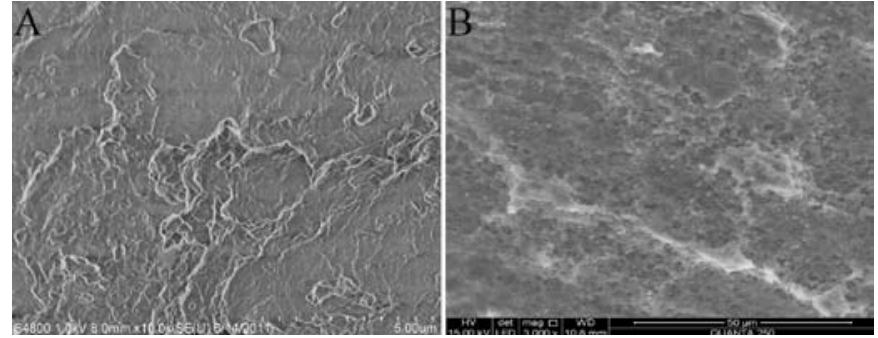

Figure 10. Degradation morphology of the NZK alloy implanted into the rabbit femur at 8 weeks using scanning electron microscopy. (A) The surface of the NZK alloy was rough with a layer of off-white degradation products. (B) Scanning electron micrograph of NZK alloy showing rough surface with copious irregular corrosion pits after removing degradation products using chromic acid.

Table III. Degradation products on the surface of the NZK alloy (4 weeks).

\begin{tabular}{lrc}
\hline Elements & Weight $\%$ & Atomic $\%$ \\
\hline Oxygen & 35.10 & 29.94 \\
Carbon & 49.46 & 56.20 \\
Nitrogen & 13.01 & 12.68 \\
Magnesium & 0.96 & 0.54 \\
Phosphorus & 0.86 & 0.36 \\
Sulfur & 0.61 & 0.26 \\
\hline
\end{tabular}

\section{Discussion}

Magnesium and its alloys have been used as implants in surgery since 1907. Lambotte (19) utilized pure magnesium plate and gilded steel nail to treat a fracture of the lower leg. The pure magnesium plate corroded rapidly in vivo, and lost its integrity 8 days after surgery, and produced a great number of gas bubbles, resulting in surgery failure. Subsequently, the studies concerning use of magnesium and its alloys as implant material gradually declined. Since the 1990s, with the development of the production technique for magnesium and its alloys, researchers have achieved a great breakthrough in controlling the corrosion resistance of magnesium and its alloys. Thus, medical application of magnesium alloy has re-gained attention.

In the present study, the neotype NZK alloy implants were implanted into the rabbit femur. At various time points, their biocompatibility were assessed by detection of ALT, CREA, $\mathrm{CK}$ and MIC levels in blood and by histological analysis of heart, liver, kidney and bone from the implant region. Also, the degradation behavior of the NZK alloy was observed via X-ray, SEM and EDS. The serum biochemistry was conducted prior to the surgery and at 1, 7, 28 and 56 days after implantation. Results from this study demonstrated that no significant difference was determined in ALT and CREA levels before and after surgery in each group $(\mathrm{P}>0.05)$. There were no significant differences in ALT and CREA levels between the NZK alloy, titanium alloy and sham-operated groups at the same time point after surgery $(\mathrm{P}>0.05)$. These results suggested that the liver and renal function were not adversely affected. On the first day after the surgery, there was a rapid increase in the CK levels $(\mathrm{P}<0.05)$, which returned to the pre-operative level 14 days after the surgery. However, no significant differences were observed between the three groups at the same time point after surgery $(\mathrm{P}>0.05)$, which suggested that the increase in CK levels after surgery is induced by surgical factors, not by implant of metal materials. In the NZK alloy group, the pre-operative MIC of the New Zealand white rabbit was $0.86 \pm 0.09 \mathrm{mmol} / \mathrm{l}$, and the MIC did not change significantly within the first day after the surgery. At 7 days, there was a remarkable increase in MIC levels compared with that prior to surgery $(\mathrm{P}<0.05)$, which returned to the pre-operative levels on the 14 days after implantation. Meanwhile, a significant difference was observed in the NZK alloy group compared with the titanium alloy group and sham-operated group at the same time point after surgery $(\mathrm{P}<0.05)$.

Increased MIC levels following implantation in the NZK alloy group may be due to two key reasons: i) following NZK alloy implantation, body fluid and blood supply was abundant surrounding the NZK alloy, so the NZK alloy began to degrade, and the magnesium ion was absorbed by blood vessel epithelial cells around the NZK alloy. Following a series of transport, the magnesium ions entered the blood circulation and were discharged via the kidney. ii) Surgery caused a local stress reaction and systematic reaction, thus stimulating magnesium ion transfer from inside cells to outside cells in order to promote wound healing. Pathological sections of heart, liver, kidney and bone from the implant region showed that the histological structure did not obviously change (Figs. 7 and 8).

The morphology of the myocardium appeared normal, and did not show substantial change. The hepatocytes were not swollen or necrotic, and the bile duct did not demonstrate notable distension and deposition. No edema or necrosis was detected in the renal glomerulus. No inflammatory cell infiltration was determined in bone from implant region. Our study suggested that NZK alloy has excellent biocompatibility as degraded biomaterial implanted into the rabbit femur. Therefore, NZK alloy could be a promising degradable material for orthopedic applications.

Magnesium and its alloys are easily corroded in solutions, especially in the presence of chloride ions. In this study, the degradation behavior of NZK alloy was observed by X-ray, SEM and EDS. According to the X-ray images, NZK alloy implants evenly degraded on 28 days following implantation, and changes of metal size were not obvious. X-ray images of the NZK alloy implants were fuzzy at 56 days after the implantation, suggesting that the material may have been gradually absorbed in vivo. The degradation of magnesium and its alloys is affected by various aspects, including chemistry, physics and electrochemistry. In vivo degradation is also affected by surrounding $\mathrm{pH}$ value and protein. Magnesium can have the following reactions in aqueous solution (20):

$$
\mathrm{Mg}+2 \mathrm{H}_{2} \mathrm{O} \rightarrow \mathrm{Mg}(\mathrm{OH})_{2}+\mathrm{H}_{2}
$$

The precise reaction procedures are as follows:

$\mathrm{Mg} \rightarrow \mathrm{Mg}^{2+}+2 \mathrm{e}^{-}$(anodic reaction) $2 \mathrm{H}_{2} \mathrm{O}+2 \mathrm{e}^{-} \rightarrow \mathrm{H}_{2}+2 \mathrm{OH}^{-}$(cathodic reaction) 


$$
\mathrm{Mg}^{2+}+2 \mathrm{OH}^{-} \rightarrow \mathrm{Mg}(\mathrm{OH})_{2}
$$

Magnesium and its alloys produce oxide film in corrosion medium. $\mathrm{Mg}(\mathrm{OH})_{2}$ is porous, and cannot effectively protect magnesium and its alloys. The speed of degradation is associated with the alloying elements, the surrounding temperature, $\mathrm{pH}$ value and negative ion concentration. Magnesium is an active metal, easily reacts with many solutions, especially chloride ion solution. $\mathrm{Cl}^{-}$can transform $\mathrm{Mg}(\mathrm{OH})_{2}$ into $\mathrm{MgCl}_{2}$, and then $\mathrm{MgCl}_{2}$ will dissolve to $\mathrm{Mg}^{2+}$ and two $\mathrm{Cl}^{-}$, which will locate on the surface of the magnesium and its alloys (10).

$$
\begin{gathered}
\mathrm{Mg}(\mathrm{OH})_{2}+2 \mathrm{Cl}^{-} \rightarrow \mathrm{MgCl}_{2} \\
\mathrm{MgCl}_{2} \rightarrow \mathrm{Mg}^{2+}+2 \mathrm{Cl}^{-}
\end{gathered}
$$

Alloying is a general approach to improve the corrosion resistance of magnesium. Therefore, in the present research, neodymium, zinc and zirconium were chosen as the alloying elements to improve the corrosion resistance of magnesium alloy. Witte et al (4) added the rare earth elements to the magnesium, and made magnesium rare earth alloys for in vivo degradation tests. In their study, while magnesium alloys LAE442 and WE43 were compared with magnesium alloys AZ31 and AZ91, LAE442 had the lowest degradation rate, indicating that the addition of alloying elements can affect the degradation rate of magnesium and its alloys. Zinc is a common alloying element added in magnesium and its strengthening effect is second only to aluminum (21). Xu et al (13) proposed that the addition of zinc in magnesium could improve the corrosion resistance. Zirconium is usually used as a grain refiner in magnesium alloys, thereby contributing to the strength of these alloys (22). Thus, it can be suggested that the corrosion resistance of our NZK alloy has been elevated by the addition of the elements neodymium, zinc and zirconium in magnesium. From Table II, it should be noted that the degradation rate of NZK alloy at 56 days implantation was lower than that at 28 days, owing to the corrosion layer on the surface of NZK alloy prohibiting the degradation. The surface of NZK alloy was covered by a layer of powder-like products at 56 days after the surgery, and the EDS analysis of the remaining materials showed that oxygen, carbon, nitrogen, magnesium and phosphorus were the main degradation products (Fig. 10A).

In summary, radiographic images showed that NZK alloy implants were gradually absorbed within 56 days when they were implanted into the femur of New Zealand white rabbits. Histology of heart, liver, kidney, and bone from the implant region was normal, and there was no infiltration by inflammatory cells, suggesting that the NZK alloy and its degradation products have excellent biocompatibility with heart, liver, kidney, and bone from the implant region. No significant difference was determined in liver and kidney function before and after surgery in each group $(\mathrm{P}>0.05)$, and there were no significant differences in liver and kidney function among NZK alloy, titanium alloy and sham-operated groups at the same time point following surgery $(\mathrm{P}>0.05)$. Results from this study demonstrated that NZK alloy has no adverse effects on liver and kidney functions, without toxic reaction, which indicate that the NZK alloy has excellent biocompatibility in vivo. The results from this study suggest that the NZK alloy can be absorbed gradually, and has excellent biocompatibility in vivo.
However, our study is limited by the investigation of shortterm degradation behavior and biocompatibility of NZK alloy in vivo. There will be a need for in vitro studies and long-term in vivo studies to validate and supplement the current results.

In conclusion, in this manuscript, the in vivo degradation behavior and biocompatibility of NZK alloy implanted into the rabbit femur have been studied and the following conclusions can be drawn: i) ZNK alloy rods were implanted into the femur of the New Zealand white rabbits, and the implants degraded by $\sim 91.5$ and $87.8 \%$ of the original weight at 28 and 56 days respectively, suggesting that the NZK alloy can be absorbed in the rabbit bone tissue gradually. ii) The implants of the NZK alloy implanted into the rabbit bone tissue had no measurable effect on ALT, CREA, CK and MIC. There were no changes in the histology of the heart, liver, kidney, and bone from the implant region postoperatively. These results suggest that NZK alloy has excellent biocompatibility in vivo.

\section{Acknowledgements}

This study was supported by the National Natural Science Foundation of China (Grant no. 81071452).

\section{References}

1. Staiger MP, Pietak AM, Huadmai J and Dias G: Magnesium and its alloys as orthopedic biomaterials: a review. Biomaterials 27: 1728-1734, 2006.

2. Witte F: The history of biodegradable magnesium implants: a review. Acta Biomater 6: 1680-1692, 2010.

3. Witte F, Feyerabend F, Maier P, Fischer J, Störmer M, Blawert C, Dietzel W and Hort N: Biodegradable magnesium-hydroxyapatite metal matrix composites. Biomaterials 28: 2163-2174, 2007.

4. Witte F, Fischer J, Nellesen J, Crostack HA, Kaese V, Pisch A, Beckmann $\mathrm{F}$ and Windhagen $\mathrm{H}$ : In vitro and in vivo corrosion measurements of magnesium alloys. Biomaterials 27: 1013-1018, 2006.

5. Witte F, Kaese V, Haferkamp H, Switzer E, Meyer-Lindenberg A, Wirth CJ and Windhagen $\mathrm{H}$ : In vivo corrosion of four magnesium alloys and the associated bone response. Biomaterials 26: 3557-3363, 2005.

6. He YH, Tao HR, Zhang Y, Jiang Y, Zhang SX, Zhao CL, Li JN, Zhang BL, Song Y and Zhang XN: Biocompatibility of bio-Mg-Zn alloy within bone with heart, liver, kidney and spleen. Chin Sci Bull 54: 484-491, 2009.

7. Ryan G, Pandit A and Apatsidis DP: Fabrication methods of porous metals for use in orthopaedic applications. Biomaterials 27: 2651-2670, 2006.

8. Toolan BC: Current concepts review: orthobiologics. Foot Ankle Int 27: 561-566, 2006.

9. Zberg B, Uggowitzer PJ and Löffler JF: MgZnCa glasses without clinically observable hydrogen evolution for biodegradable implants. Nat Mater 8: 887-891, 2009.

10. Li Z, Gu X, Lou S and Zheng Y: The development of binary $\mathrm{Mg}-\mathrm{Ca}$ alloys for use as biodegradable materials within bone. Biomaterials 29: 1329-1344, 2008.

11. Wei J, Jia J, Wu F, Wei S, Zhou H, Zhang H, Shin JW and Liu C: Hierarchically microporous/macroporous scaffold of magnesiumcalcium phosphate for bone tissue regeneration. Biomaterials 31 : 1260-1269, 2010.

12. Xu L, Pan F, Yu G, Yang L, Zhang E and Yang K: In vitro and in vivo evaluation of the surface bioactivity of a calcium phosphate coated magnesium alloy. Biomaterials 30: 1512-1523, 2009.

13. Xu L, Zhang E, Yin D, Zeng S and Yang K: In vitro corrosion behaviour of $\mathrm{Mg}$ alloys in a phosphate-buffered solution for bone implant application. J Mater Sci Mater Med 19: 1017-1025, 2008.

14. Zhang SX, Li JN, Song Y, Zhao CL, Zhang XN, Xie CY, Zhang Y, Tao HR, He YH, Jiang Y and Bian YJ: In vitro degradation, hemolysis and MC3T3-E1 cell adhesion of biodegradable Mg-Zn alloy. Mat Sci Eng C 29: 1907-1912, 2009. 
15. Zhang SX, Zhang XN, Zhao CL, Li JN, Song Y, Xie CY, Tao HR, Zhang Y, He YH, Jiang Y and Bian YJ: Research on an Mg-Zn alloy as a degradable biomaterial. Acta Biomater 6: 626-640, 2010.

16. Kirkland NT, Birbilis N, Walker J, Woodfield T, Dias GJ and Staiger MP: In vitro dissolution of magnesium-calcium binary alloys: clarifying the unique role of calcium additions in bioresorbable magnesium implant alloys. J Biomed Mater Res B Appl Biomater 95: 91-100, 2010.

17. Yang J, Cui F and Lee IS: Surface modifications of magnesium alloys for biomedical applications. Ann Biomed Eng 39: 1857-1871, 2011.

18. Witte F, Fischer J, Nellesen J, Vogt C, Vogt J, Donath T and Beckmann F: In vivo corrosion and corrosion protection of magnesium alloy LAE442. Acta Biomater 6: 1792-1799, 2010.
19. Lambotte A: L'utilisation du magnesium comme materiel perdu dans l'osteosynthese. Bull Mem Soc Nat Chir 28: 1325-1334, 1932 (In French).

20. Witte F, Hort N, Vogt C and, et al: Degradable biomaterials based on magnesium corrosion. Curr Opin Solid State Mater Sci 12: 63-72, 2008.

21. Boehlert CJ and Knittel K: The microstructure, tensile properties, and creep behaviour of Mg-Zn alloys containing 0-4.4 wt.\% Zn. Mater Sci Eng A 417: 315-321, 2006.

22. Babkin VM: Effect of zirconium on the grain size of magnesium containing 4.5\% Zn. Met Sci Heat Treat 5: 543-544, 2004. 